\title{
Hedyotis diffusa willd extract suppresses colorectal cancer growth through multiple cellular pathways
}

\author{
JIANYU FENG $^{1,2}$, YIYI JIN ${ }^{1,2}$, JUN PENG ${ }^{1,2}$, LIHUI WEI ${ }^{1,2}$, QIAOYAN CAI ${ }^{1,2}$, \\ ZHAOKUN YAN $^{1,2}$, ZIJUN LAI ${ }^{1,2}$ and JIUMAO LIN ${ }^{1,2}$ \\ ${ }^{1}$ Biomedical Research Center of Academy of Integrative Medicine of Fujian University of \\ Traditional Chinese Medicine; ${ }^{2}$ Fujian Key Laboratory of Integrative Medicine on Geriatrics, \\ Fujian University of Traditional Chinese Medicine, Fuzhou, Fujian 350122, P.R. China
}

Received April 29, 2016; Accepted July 21, 2017

DOI: $10.3892 / 01.2017 .7244$

\begin{abstract}
The development of colorectal cancer (CRC) is strongly associated with the imbalance of various intracellular signal transduction cascades, including protein kinase B (AKT), mitogen-activated protein kinase 1 (MAPK), signal transducer and activator of transcription 3 (STAT3), as well as crosstalk between these signaling networks. At present, anti-tumor agents are often single-targeted and therefore are not always therapeutically effective. Moreover, long-term use of these anti-tumor agents often generates drug resistance and potential side effects. These problems highlight the urgent need for the development of novel and more effective anti-cancer drugs. Hedyotis diffusa Willd (HDW) has been used as a major component in traditional Chinese medicine for the clinical treatment of colorectal cancer, with a limited number of adverse effects. However, the molecular mechanisms, which underlie its anti-cancer activity, still require further elucidation. In the present study, using xenograft models and various different human CRC cell lines, the efficacy of the ethanol extract of HDW (EEHDW) against tumor growth was evaluated, and its underlying molecular mechanisms of action were investigated. It was demonstrated that EEHDW was able to inhibit cancer growth in vivo and in vitro. Furthermore, EEHDW was able to suppress the activation of several CRC-associated signaling pathways and was able to regulate the expression of various inflammatory and angiogenic factors. This resulted in the induction of apoptosis and inhibition of cellular proliferation,
\end{abstract}

Correspondence to: Dr Jiumao Lin, Biomedical Research Center of Academy of Integrative Medicine of Fujian University of Traditional Chinese Medicine, 1 Qiuyang Road, Minhou Shangjie, Fuzhou, Fujian 350122, P.R. China

E-mail: jiumaolin@hotmail.com

Abbreviations: CRC, colorectal cancer; EEHDW, ethanol extract of Hedyotis diffusa Willd; TCM, traditional Chinese medicine

Key words: colorectal cancer, Hedyotis diffusa Willd, signaling pathway, cytokine as well as tumor angiogenesis. The present study demonstrated that EEHDW is able to exhibit anti-cancer activity due to its ability to affect multiple intracellular targets, which suggests that it may be a novel multi-potent therapeutic agent for the treatment of colorectal cancer.

\section{Introduction}

Colorectal cancer (CRC) is the third most common type of cancer affecting the gastrointestinal tract worldwide (1). CRC has a high mortality rate and is often associated with rapid cancer progression and advanced tumor presentation at the time of diagnosis $(2,3)$. Recent changes in lifestyle, diet and an aging population have all contributed to the increased prevalence of CRC in China (4). Patients who have been diagnosed with early stage CRC frequently are treated with surgery in conjunction with chemotherapy and radiotherapy to improve their survival rate (1). However, for the majority of patients diagnosed with CRC have already reached an advanced pathological stage, chemotherapy is the major form of treatment (5-8). In particular, 5-fluorouracil (5-FU)-based regimens are used as the international standard for late-stage CRC treatment $(9,10)$. Nevertheless, the increasing occurrence of multi-drug resistance (MDR) has had negative impact on the clinical treatment of CRC $(11,12)$. Other currently used anti-cancer agents often present unacceptable levels of toxicity to normal cells and tissues $(13,14)$, thereby limiting their effectiveness.

The development of colorectal cancer is a complex and multi-stage process, characterized by various interactions between environmental carcinogens, genetic modifications and the host immune system (15). Dense infiltration of cytokine-producing immune/inflammatory cells frequently result in the aberrant activation of multiple intracellular signal transduction cascades, ultimately resulting in the uncontrolled growth of transformed cells. In addition, crosstalk between these signaling pathways results in a complicated and robust cellular signal transduction network that is regulated by compensatory mechanisms. Therefore, anti-cancer drugs that target only a single signaling pathway might not be as effective and is more prone to the development of MDR or adverse side effects (16). 
Hedyotis diffusa Willd (HDW) is a medicinal herb, which belongs to the Rubiaceae family, and is widely distributed in Northeast Asia (17). It is a well-known traditional Chinese herbal medicine used for 'heat-clearing', detoxification and promoting blood circulation (18). HDW has also been widely used as an important component in traditional Chinese medicine (TCM) prescription formulae for the treatment of various types of cancer, including CRC (17-19). Our previous studies have demonstrated that HDW is able to inhibit colon cancer growth in vivo and in vitro by promoting apoptosis of cancer cells, inhibiting proliferation, tumor angiogenesis and MDR reversal (20-25). However, the precise mechanism of the in vivo anti-cancer activity of HDW remains largely unknown. In the present study, the present authors evaluated the efficacy of HDW in inhibiting CRC growth in a mouse xenograft model as well as various CRC cell lines in order to further elucidate the anti-cancer mechanism of HDW.

\section{Materials and methods}

Materials and reagents. RPMI-1640, Dulbecco's modified Eagle's medium (DMEM) with high glucose, Fetal bovine serum (FBS), penicillin-streptomycin (catalog no. SV30010), $0.25 \%$ trypsin-EDTA, Pierce RIPA buffer, Pierce BCA Protein Assay kit (catalog no. 23227), and SuperSignal ${ }^{\mathrm{TM}}$ West Pico Chemiluminescent substrate (catalog no. 34080) were all purchased from Thermo Fisher Scientific, Inc. (Waltham, MA, USA). CellTiter $96^{\circledR}$ AQueous Non-Radioactive Cell Proliferation Assay kit (MTS assay; catalog no. G5430) was provided by Promega Corporation (Madison, WI, USA). Matrigel was obtained from BD Biosciences (Franklin Lakes, NJ, USA). TumorTACS in situ Apoptosis kit (catalog no. 4815-30-K) was purchased from R\&D Systems Inc. (Minneapolis, MN, USA). VECTASTAIN Elite ABC kit was provided by Vector Laboratories, Inc. (Burlingame, CA, USA). Rabbit monoclonal antibodies against Pim-1 (catalog no. ab75776), B-cell lymphoma 2 (Bcl-2; catalog no. ab32124), cyclooxygenase-2 (COX-2; catalog no. ab62331), inducible nitric oxide synthase (iNOS; catalog no. ab178945), endothelial nitric oxide synthase (eNOS; catalog no. ab66127), rabbit polyclonal antibody against Ki-67 (catalog no. ab15580), Bcl-2-like protein 4 (Bax; catalog no. ab69643) and mouse monoclonal antibody against hypoxia-inducible factor 1- $\alpha$ (HIF1- $\alpha$; catalog no. ab463) were purchased from Abcam (Cambridge, MA, USA). Rabbit monoclonal antibodies against cytochrome $C$ (catalog no. 4280), caspase-3 (catalog no. 9665), poly (ADP-ribose) polymerase 1 (PARP; catalog no. 9532), mouse monoclonal antibody against caspase-9 (catalog no. 9508), and rabbit polyclonal antibody against $\beta$-actin (catalog no. 4967) were obtained from Cell Signaling Technology, Inc., (Danvers, MA, USA). Horseradish peroxidase (HRP)-conjugated goat anti-rabbit immunoglobulin G (IgG; catalog no. E030120) and goat anti-mouse IgG secondary antibodies (catalog no. E030110) was purchased from Earthox, LLC (Millbrae, CA, USA). Mouse interleukin (IL)-1 $\beta$, IL-6, IL-4, IL-10 and tumor necrosis factor (TNF)- $\alpha$ ELISA kits were obtained from Shanghai Westang Bio-tech Co., Ltd. (Shanghai, China). Bio-Plex Phosphoprotein Detection Reagent kit (catalog no. 171-304005) were purchased from Bio-Rad Laboratories (Hercules, CA, USA). Culture flask and plates were purchased from Wuxi NEST Biotechnology Co., Ltd. (Wuxi, Jiangsu, China). All the other chemicals used, unless otherwise stated, were obtained from Sigma-Aldrich (Merck KGaA, Darmstadt, Germany).

Preparation of ethanol extract from Hedyotis diffusa Willd $(E E H D W)$. Authentic plant material was purchased from Guo Yi Tang Chinese herbal medicine store (Fujian, China). Identification of the Hedyotis diffusa Willd (HDW) herb was confirmed by Dr Wei Xu (Department of Pharmacology, Fujian University of Traditional Chinese Medicine, Fujian, China). Ethanol extract of HDW (EEHDW) was prepared as previously described (20-22). Briefly, 500 g HDW was extracted with $5,000 \mathrm{ml}$ of $85 \%$ ethanol using refluxing method and then filtered. The ethanol solvent was then evaporated on a rotary evaporator. The resultant solution was concentrated to a relative density of 1.05, and the dried powder of EEHDW was obtained using spray desiccation method using a spray dryer.

For in vitro experiments, stock solutions of EEHDW were prepared by dissolving the EEHDW powder in $40 \%$ dimethyl sulfoxide (DMSO) to achieve a final concentration of $500 \mathrm{mg} / \mathrm{ml}$. Working concentrations of EEHDW were prepared by diluting the stock solution in culture medium (RPMI-1640 for HCT-8 and HCT-116; DMEM for HT-29 and SW620). The final concentration of DMSO in the medium was $<0.2 \%$. For in vivo experiments, working concentrations of EEHDW were prepared by dissolving the EEHDW powder in saline to a concentration of $0.1 \mathrm{~g} / \mathrm{ml}$.

Cell culture. Human CRC cell lines, HCT-8, HT-29, HCT-116 and SW620, were purchased from Cell Bank of the Chinese Academy of Sciences (Shanghai, China). HCT-8 and HCT-116 cells were cultured in RPMI-1640. HT-29 and SW620 cells were cultured in DMEM. All cell media were supplemented with $10 \%$ (v/v) FBS, $100 \mathrm{U} / \mathrm{ml}$ penicillin and $100 \mu \mathrm{g} / \mathrm{ml}$ streptomycin and cultured at $37^{\circ} \mathrm{C}$, with $5 \% \mathrm{CO}_{2}$ in a humidified incubator.

Evaluation of cell viability. Cell viability was assessed using MTS assay. The cells $(10,000 /$ well $)$ were incubated in 96-well plates with culture medium at $37^{\circ} \mathrm{C}$ for $12 \mathrm{~h}$ and then treated with various concentrations of EEHDW $(0,0.5,1$ and $2 \mathrm{mg} / \mathrm{ml})$ for 24 and $48 \mathrm{~h}$; the control cells were treated with DMSO without EEHDW at $37^{\circ} \mathrm{C}$ for 24 and $48 \mathrm{~h}$. Next, $10 \mu \mathrm{l}$ MTS was added to each well, and the samples were incubated for $1 \mathrm{~h}$ at $37^{\circ} \mathrm{C}$. The resulting absorbance was measured at $490 \mathrm{~nm}$ using an ELISA reader (BioTek Instruments, Inc., Winooski, VT, USA).

Animals. A total of 20 -week-old athymic BALB/c nu/nu male mice (initial body weight, $20 \pm 2 \mathrm{~g}$ ) were obtained from Shanghai SLAC Laboratory Animal Co., Ltd. (Shanghai, China) and housed under pathogen-free conditions $\left(22^{\circ} \mathrm{C}\right.$; 12-h light/dark cycle). Food and water were given ad libitum throughout the experiment. All animal experiments were approved by the Institutional Animal Care and Use Committee of Fujian University of Traditional Chinese Medicine (Fujian, China).

In vivo nude mouse xenograft study. HT-29 cells $\left(5 \times 10^{6}\right)$ mixed with Matrigel (1:1) were subcutaneously injected in 
the right flank area of athymic nude mice to initiate tumor growth. After 5 days of xenograft implantation, the mice were randomly divided into two groups $(n=10)$ and given daily intra-gastric administration with $1 \mathrm{~g} / \mathrm{kg}$ EEHDW or saline for 6 days per week for a total of 16 days. The diameters of the tumors were measured every second day with digital calipers and the tumor volume (V) was calculated using the formula: $\mathrm{V}=(\text { width })^{2} \mathrm{x}$ length $\mathrm{x} \pi / 6$. At the end of experiment, the mice were sacrificed using $100 \mathrm{mg} / \mathrm{kg}$ pelltobarbitalum natricum (Sigma-Aldrich; Merck KGaA), and the tumor tissues were removed and fixed in $4 \%$ paraformaldehyde (China National Medicines Corporation Ltd., Beijing, China) or stored at $-80^{\circ} \mathrm{C}$. Blood was collected aseptically from the orbital sinus. Blood collection tubes were allowed to stand at room temperature for $5 \mathrm{~h}$ prior to serum collection by centrifugation at $2,000 \mathrm{x} \mathrm{g}$ for $20 \mathrm{~min}$ at room temperature, and then stored at $-80^{\circ} \mathrm{C}$.

Immunohistochemical (IHC) staining. Tumor tissues were analyzed by IHC as previously described (24). Briefly, following fixation at room temperature with $4 \%$ paraformaldehyde for $24 \mathrm{~h}$, the tumor samples were paraffin-embedded using $100 \%$ liquid paraffin at $56^{\circ} \mathrm{C}$ until the paraffin had fully hardened, and sliced into $4 \mu \mathrm{m}$-thick sections. The slides were subjected to antigen retrieval and endogenous peroxidase activity was quenched using hydrogen peroxide. IHC staining was performed using the VECTASTAIN Elite $\mathrm{ABC}$ kit according to the manufacturer's instructions. Briefly, following blocking of non-specific proteins with normal serum in PBS (0.1\% Tween 20), the slides were incubated with $\mathrm{Ki}-67$, Pim-1, Bcl-2, Bax, COX-2, iNOS, eNOS, HIF1- $\alpha$ (all 1:200 diluted in PBS) primary antibodies or PBS (negative control) overnight at $4^{\circ} \mathrm{C}$. After washing with PBS, the slides were incubated for $30 \mathrm{~min}$ at room temperature with biotinylated secondary antibody (goat anti-rabbit immunoglobulin G; cat. no. E030120) followed by conjugated HRP-labeled streptavidin (Dako; Agilent Technologies, Inc., Santa Clara, CA, USA) and then washed with PBS. The slides were then incubated with 3,3'-diaminobenzidine (DAB, Sigma-Aldrich, Merck KGaA) as the chromogen, followed by counterstaining with diluted hematoxylin (Sigma-Aldrich, Merck KGaA) at room temperature for $30 \mathrm{sec}$. After staining, five high-power fields (magnification, x400) were randomly selected in each slide and the mean proportion of positive cells in each field were counted using the true color multi-functional cell image analysis management system (version 6.0; Image-Pro Plus; Media Cybernetics, Inc., Rockville, MD, USA).

In situ apoptosis detection by terminal deoxynucleotidyl-transferase-mediated dUTP nick end labeling (TUNEL) staining. Apoptosis in tumor tissues were analyzed by TUNEL staining using TumorTACS in situ Apoptosis kit as described previously (22). Briefly, apoptotic cells were examined by light microscopy (DM4000, Leica Microsystems GmbH, Wetzlar, Germany) and counted as DAB-positive cells (brown staining) at five arbitrarily selected microscopic fields (magnification, $\mathrm{x} 400$ ). TUNEL-positive cells were counted as a percentage of the total cells.

Preparation of tumor homogenate and western blotting. A total of three tumors were randomly selected from the control and the EEHDW group, washed with PBS 3 times and homogenized in Pierce RIPA buffer containing protease inhibitor and phosphatase inhibitor cocktails. The samples were then centrifuged at $17,000 \mathrm{x}$ g for $20 \mathrm{~min}$ at $4^{\circ} \mathrm{C}$, and the resulting protein concentrations were determined using BCA Protein Assay reagent kit. A total of $50 \mu \mathrm{g}$ protein for each sample was loaded onto $10 \%$ SDS-PAGE and resolved at $20 \mathrm{~V}$ for $10 \mathrm{~min}$, at $80 \mathrm{~V}$ for $30 \mathrm{~min}$ and at $120 \mathrm{~V}$ for $1 \mathrm{~h}$. The proteins were then transferred onto nitrocellulose membranes. Following blocking with $5 \%$ non-fat milk powder at room temperature for $2 \mathrm{~h}$ with the $5 \%$ non-fat milk powder dissolved using TBS with Tween-20 (TBST, pH8.0) containing 0.1\% Tween, the membranes were incubated with cytochrome C, caspase-3, caspase-9, PARP and $\beta$-actin (all 1:1,000) primary antibodies overnight at $4^{\circ} \mathrm{C}$, and then incubated with the aforementioned HRP-conjugated anti-rabbit secondary antibodies to bind the antibodies of cytochrome $\mathrm{C}$, caspase-3, PARP and $\beta$-actin, or anti-mouse secondary antibody to bind the caspase- 9 antibody (all 1:5,000) for $1 \mathrm{~h}$ at room temperature. The membranes were then subjected to enhanced chemiluminescence (ECL) detection using SuperSignal ${ }^{\mathrm{TM}}$ West Pico Chemiluminescent substrate. Image $\mathrm{Lab}^{\mathrm{TM}}$ software (version 3.0; Bio-Rad Laboratories, Inc.) was used for densitometric analysis.

Measurement of cytokines in serum by ELISA. Blood were collected and stored at $-80^{\circ} \mathrm{C}$ until further analysis. The level of IL-1 $\beta$, IL-6, IL-4, IL-10 and TNF- $\alpha$ in the serum was measured using IL-1 $\beta$ (cat. no. F10770), IL-6 (cat. no. F10830), IL-4 (cat. no. F10810), IL-10 (cat. no. F10870) and TNF- $\alpha$ (cat. no. F11630) ELISA kits (Xitang Biological Technology Co., Ltd., Shanghai, China), respectively, according to the manufacturer's protocol. Briefly, $100 \mu \mathrm{l}$ diluted standard and test samples were added in each well. Plates were covered and incubated for $2 \mathrm{~h}$ at $37^{\circ} \mathrm{C}$, then washed 5 times prior to incubation with $50 \mu \mathrm{l}$ biotinylated antibody solution for $1 \mathrm{~h}$ at $37^{\circ} \mathrm{C}$. The Plates were washed three times, and $100 \mu 1$ streptavidin-HRP solution was distributed to all wells prior to incubation for $1 \mathrm{~h}$ at $37^{\circ} \mathrm{C}$. After washing, the substrate was added and incubated for $15 \mathrm{~min}$ at room temperature in a darkened room. Finally, the reactions were stopped and the absorbance was measured at $450 \mathrm{~nm}$. Wells with no biotinylated antibody solutions or streptavidin-HRP solution were used as the negative controls. The concentrations of the aforementioned cytokines were determined by comparing to serial dilutions of the purified standards.

Bio-Plex phosphoprotein assay. A total of 8 tumors were randomly selected from the EEHDW and control groups, and homogenized. For analysis of phosphorylation of protein kinase B (AKT), mitogen-activated protein kinase 1/2 (Erk1/2), c-Jun N-terminal kinase (JNK), p38, p53 and p70S6K in vitro, HT-29 cells $\left(2.5 \times 10^{5}\right)$ were seeded into $25 \mathrm{~cm}^{2}$ flasks with $5 \mathrm{ml}$ RPMI-1640 medium and treated with $2 \mathrm{mg} / \mathrm{ml}$ EEHDW for $24 \mathrm{~h}$; the control cells were treated with RPMI-1604 with $0.16 \%$ DMSO without EEHDW at $37^{\circ} \mathrm{C}$ for $24 \mathrm{~h}$. To detect signal transducer and activator of transcription 3 (STAT3) phosphorylation in vitro, HT-29 cells were first cultured at $37^{\circ} \mathrm{C}$ in complete DMEM (10\% FBS) until $70 \%$ confluence, and subsequently cultured at $37^{\circ} \mathrm{C}$ in FBS-free medium overnight. The medium was replaced with DMEM with $10 \%$ FBS, 

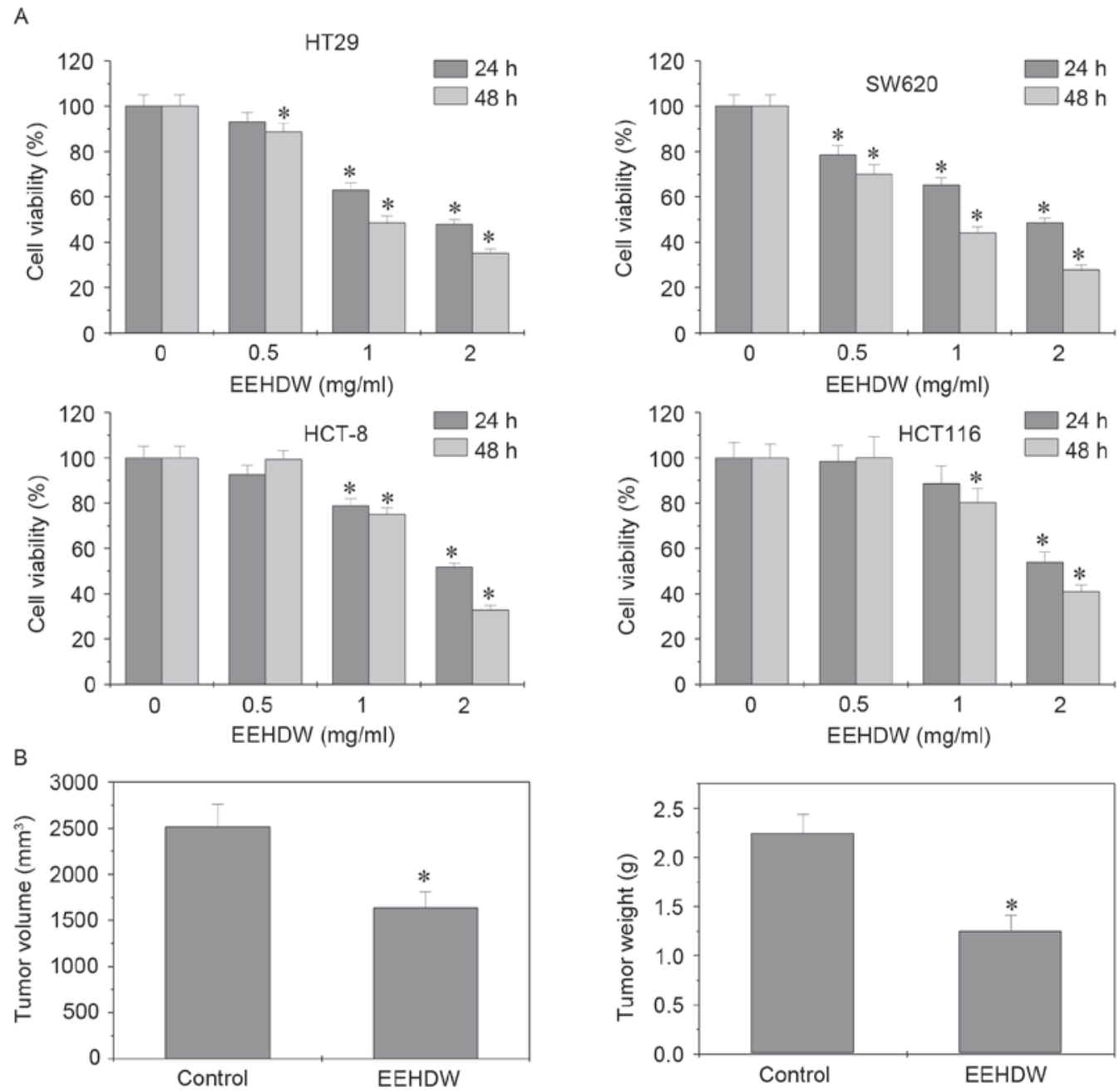

Figure 1. EEHDW inhibits growth of colorectal cancer cells and xenograft tumors in mice. (A) The cells were treated with various concentrations of EEHDW $(0,0.5,1$ and $2 \mathrm{mg} / \mathrm{ml})$ for 24 and $48 \mathrm{~h}$. Cell viability was assessed using MTS assay. Data are shown as the mean \pm standard deviation from three independent experiments. ${ }^{*} \mathrm{P}<0.05$ vs. untreated control cells. (B) The mice were administered intragastrically with $1 \mathrm{~g} / \mathrm{kg}$ EEHDW or saline for 6 days per week for a total of 16 days. Tumor weight and volume were measured at the end of the experiment. Data are expressed as the mean \pm standard deviation from 10 individual mice in each group. " $\mathrm{P}<0.01$ vs. control mice. EEHDW, ethanol extract of Hedyotis Diffusa Willd.

and the cells were pre-treated with EEHDW $(2 \mathrm{mg} / \mathrm{ml})$ for $1 \mathrm{~h}$ at $37^{\circ} \mathrm{C}$, followed by stimulation with $10 \mathrm{ng} / \mathrm{ml} \mathrm{IL}-6$ for $15 \mathrm{~min}$ at $37^{\circ} \mathrm{C}$. Tumor tissues and treated cells were lysed using a commercially available lysis kit (Bio-Rad Laboratories) and centrifuged at $17,000 \mathrm{x}$ g for $20 \mathrm{~min}$ at $4^{\circ} \mathrm{C}$, and the resulting protein concentrations were determined by BCA protein assay. The presence of phosphorylated (p-)AKT, p-Erk1/2, p-JNK, p-p38, p-p53, p-p70S6K and p-STAT3 were detected using a bead-based multiplex assay for phosphoproteins (Bio-Plex Phosphoprotein assay kit; Bio-Rad Laboratories), according to the manufacturer's instructions. Data were collected and analyzed using the Bio-Plex 200 suspension array system (Bio-Rad Laboratories).

Statistical analysis. All data were expressed as the mean \pm standard deviation. Statistical analysis was performed using the SPSS software (version 17.0) for Windows (SPSS, Inc. Chicago, IL, USA) using one-way analysis of variance. Fisher's least significant difference (for equal variances) or Dunnett's correction (for unequal variances) were used in post-hoc tests. $\mathrm{P}<0.05$ was considered to be statistically significant.

\section{Results}

EEHDW inhibits the growth of CRC cells and xenograft tumors in mice. The effect of EEHDW on the viability of various CRC cell lines was determined using MTS assay. EEHDW treatment at $0.5,1$ and $2 \mathrm{mg} / \mathrm{ml}$ for 24 or $48 \mathrm{~h}$ was able to significantly reduce the viability of HT-29, SW620, HCT-8 and HCT-116 cells) in a dose- and time-dependent manner compared with the control (Fig. 1A). The in vivo anti-tumor effect of EEHDW was subsequently determined by comparing the tumor weight and volume between EEHDW-treated and control mice. EEHDW-treated mice exhibited a $34.94 \%$ decrease in tumor volume (control, 2,516 $\pm 245 \mathrm{~mm}^{3}$; EEHDW-treated, $\left.1,637 \pm 173 \mathrm{~mm}^{3} ; \mathrm{P}<0.0037\right)$ and a $44.14 \%$ decrease in tumor weight (control, 2.25 $\pm 0.19 \mathrm{~g}$; EEHDW-treated, m1.25 $\pm 0.16 \mathrm{~g}$; $\mathrm{P}<0.0012$ ) compared with the control mice (Fig. 1B). These results suggest that EEHDW was able to suppress colorectal tumor growth in vivo and in vitro.

EEHDW inhibits cellular proliferation and induces apoptosis in xenograft tumors. The in vivo effect of EEHDW on 
A
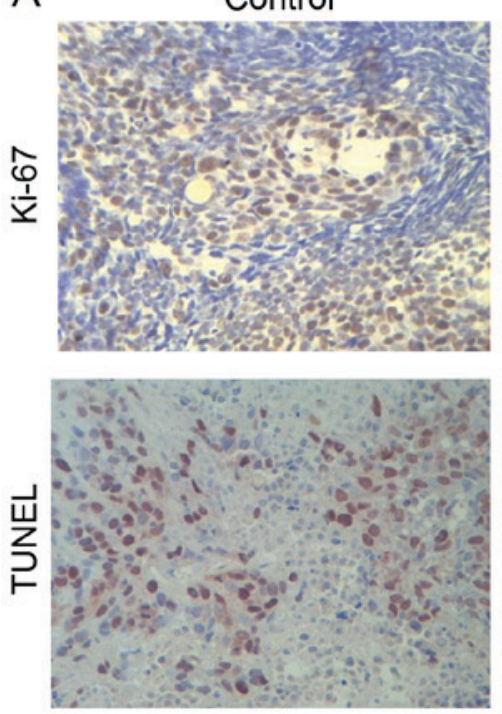

\section{EEHDW}
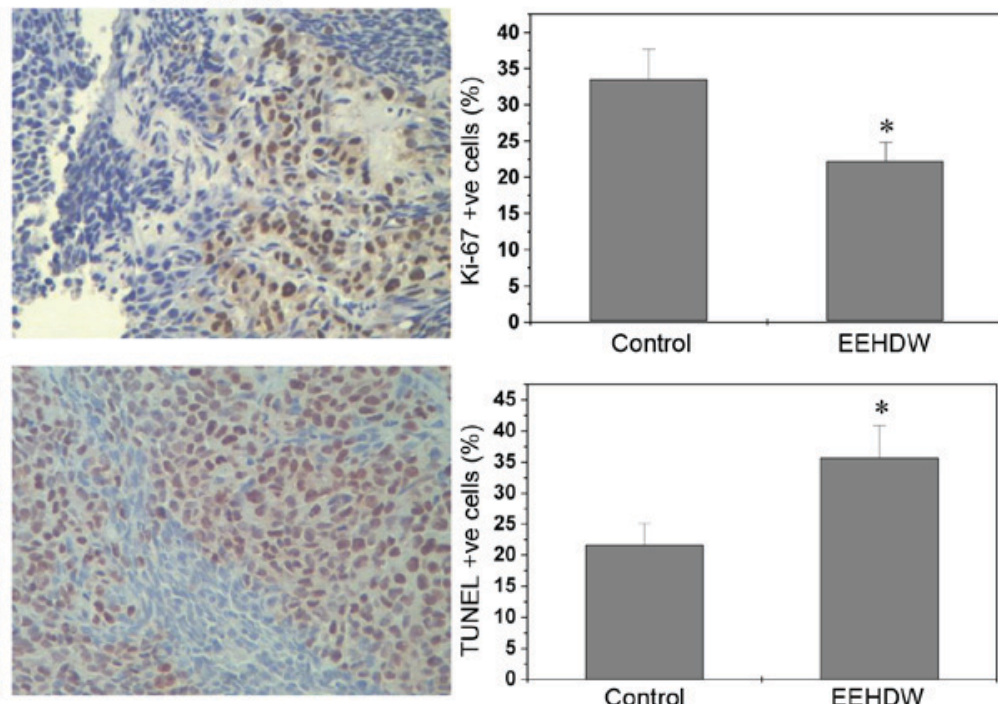

B
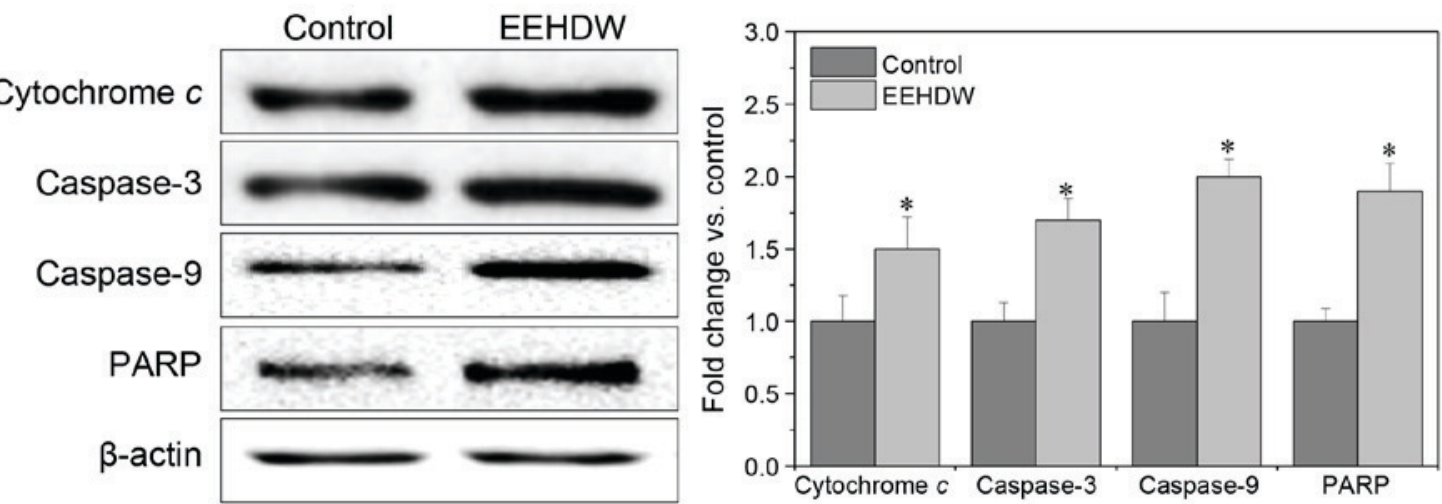

Figure 2. EEHDW inhibits cellular proliferation and induces apoptosis in xenograft tumors. (A) Staining for Ki-67 and TUNEL were performed to examine the in vivo effect of EEHDW on proliferation and apoptosis. The photographs are representative images captured at a magnification of $x 400$. The percentage of positively stained cells was also quantified. To rule out any non-specific staining, PBS was used to replace the primary antibody as a negative control. Data are expressed as the mean \pm standard deviation from 10 individual mice in each group. ${ }^{*} \mathrm{P}<0.05$ vs. control mice. (B) A total of three tumors were randomly selected from each group, and the level of cytochrome C, caspase-3, -9 and PARP in tumor tissues was determined by western blotting to examine the in vivo effect of EEHDW on apoptosis. $\beta$-actin was used as the internal control. For each tumor sample, western blotting was performed in triplicate. In densitometric analysis, the expression of the target proteins was normalized to the mean protein expression of control. " P $<0.05$, vs. control mice. EEHDW, ethanol extract of Hedyotis Diffusa Willd. TUNEL, terminal deoxynucleotidyl-transferase-mediated dUTP nick end labeling; PARP, poly(ADP-ribose)polymerase 1.

proliferation and apoptosis in mice was determined using Ki-67 and TUNEL staining, as well as western blotting for proteins in the mitochondrial cytochrome c release-mediated caspase cascade. There was a significant decrease in the number of Ki-67-positive cells in the EEHDW-treated group compared with the control group $(22.20 \pm 2.63 \%$ vs. $33.50 \pm 4.22 \% ; \mathrm{P}<0.05)$, while the percentage of TUNEL-positive cells were increased in the EEHDW-treated group compared with the control group $(35.60 \pm 5.34 \%$ vs $21.67 \pm 3.47 \%$; Fig. $2 \mathrm{~A}, \mathrm{P}<0.05)$. Additionally, the expression of cytochrome c, caspase-3, caspase-9 and PARP were upregulated in the EEHDW-treated group compared with the control group ( $\mathrm{P}<0.05$; Fig. 2B). Taken together, these results demonstrated that EEHDW was able to inhibit proliferation and promote apoptosis in vivo.

EEHDW regulates the expression of Pim-1, Bcl-2, Bax and angiogenic factors COX-2, iNOS, eNOS and HIF-1 $\alpha$. To further investigate how EEHDW is able to inhibit tumor growth, IHC staining was performed to detect the expression of Pim-1, Bcl-2 and Bax in mice. The percentage of Pim-1, Bcl-2 or Bax-positive cells in the control group was, $37.71 \pm 7.31,27.33 \pm 3.67$ and $18.50 \pm 3.83 \%$ respectively, whereas in EEHDW-treated mice the percentage was $21.75 \pm 4.57,16.00 \pm 4.31$ and $35.00 \pm 4.32 \%$, respectively $(\mathrm{P}<0.05$; Fig. 3). These data suggested that that EEHDW treatment was able to significantly $(\mathrm{P}<0.02)$ downregulate the expression of Pim-1, a potential oncogene, while decreasing Bcl-2 expression and increasing Bax expression, demonstrating that EEHDW inhibits cell proliferation and induces apoptosis in xenograft tumors. In addition, the percentage of COX-2, iNOS, eNOS or HIF-1 $\alpha$-positive cells in the control group was $33.00 \pm 5.67,45.33 \pm 6.93,30.33 \pm 5.87$, and $28.50 \pm 4.77 \%$, respectively, whereas in EEHDW-treated mice the percentage was $23.75 \pm 4.12,27.25 \pm 4.24,20.25 \pm 3.78$ and $17.00 \pm 2.86 \%$. These results suggested that EEHDW treatment was also able to downregulate the expression of COX-2, iNOS, eNOS and HIF-1 $\alpha(\mathrm{P}<0.05$; Fig. 3), which are novel tumor markers and are hypothesized to have important roles during tumor angiogenesis (26). 

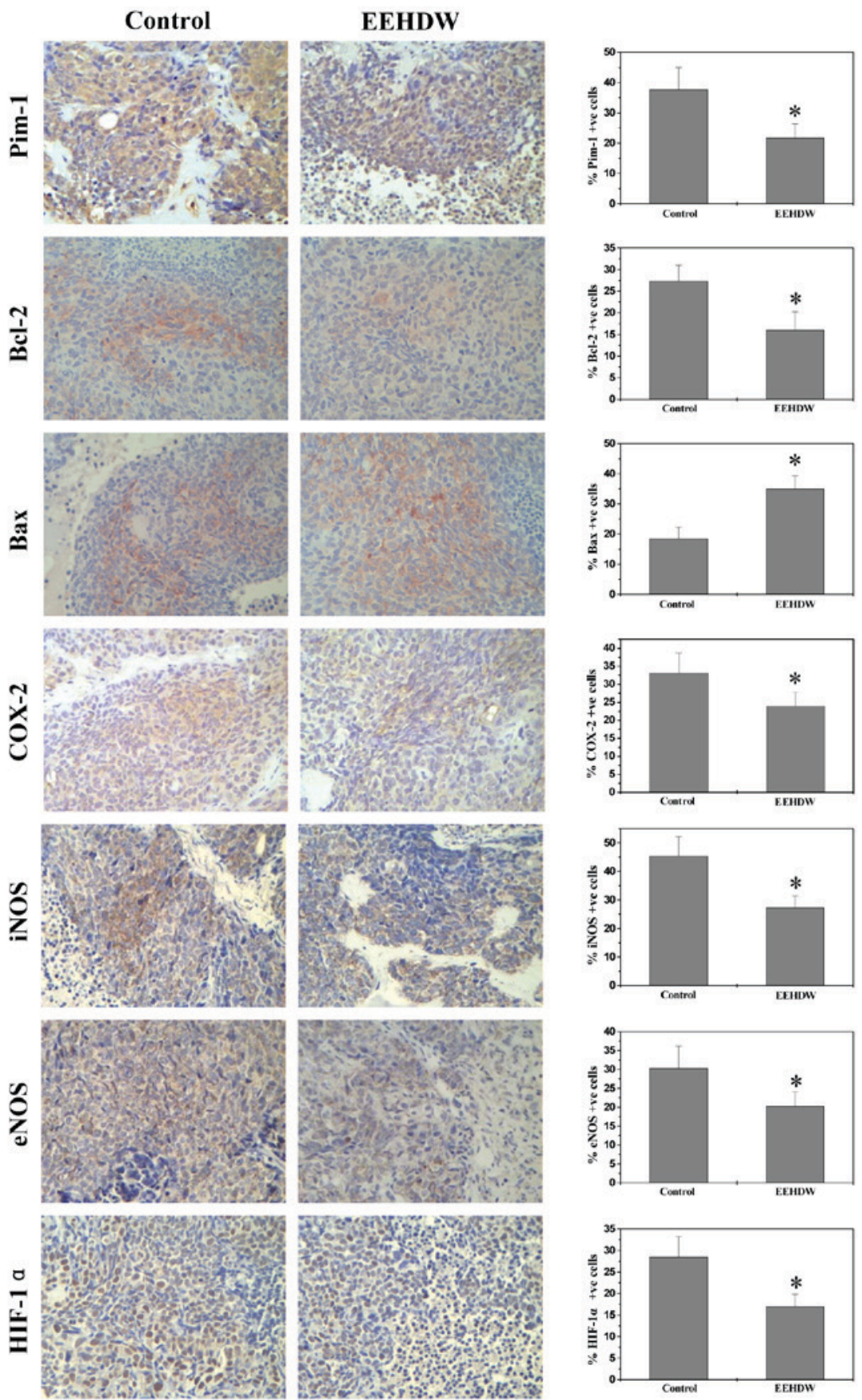

Figure 3. EEHDW regulates the expression of Pim-1, Bcl-2, Bax and angiogenic factors COX-2, iNOS, eNOS and HIF-1 $\alpha$. Tumor tissues were processed for immunohistochemical staining of Pim-1, Bcl-2, Bax, COX-2, iNOS, eNOS and HIF-1 $\alpha$. The photographs are representative images taken at a magnification of $x 400$. The percentage of positively stained cells was also quantified. Data are expressed as the mean \pm standard deviation from 10 mice in each group. ${ }^{*} \mathrm{P}<0.05$ vs. control mice. EEHDW, ethanol extract of Hedyotis Diffusa Willd; Bcl-2, B-cell lymphoma 2; Bax, Bcl-2-like protein 4; COX-2, cyclooxygenase-2; iNOS, inducible nitric oxide synthase; eNOS, endothelial nitric oxide synthase; HIF1- $\alpha$, hypoxia-inducible factor 1- $\alpha$.

EEHDW regulates inflammatory cytokines in serum. Dense infiltration of cytokine-producing immune cells is frequently observed in cancer tissues: Each immune cell subset and cytokine involved in the activation of intracellular pathways sustains the growth of cancer cells (27). The effect of EEHDW treatment on secreting cytokines was detected using ELISA, whereby the levels of IL-1 $\beta$, IL- 6 and TNF- $\alpha$ were significantly $(\mathrm{P}<0.026)$ decreased following EEHDW treatment, By contrast, the levels of IL-4 and IL-10 were upregulated following EEHDW treatment compared with the control group ( $\mathrm{P}<0.05$; Fig. 4).

EEHDW regulates multiple signaling pathways in vivo and in vitro. To investigate the underlying mechanisms of EEHDW in inhibiting tumor growth, the effect of EEHDW on the activation of various CRC-associated signal transduction cascades was determined. The effect of EEHDW treatment on the activation and phosphorylation of AKT, Erk1/2, JNK, p38, 

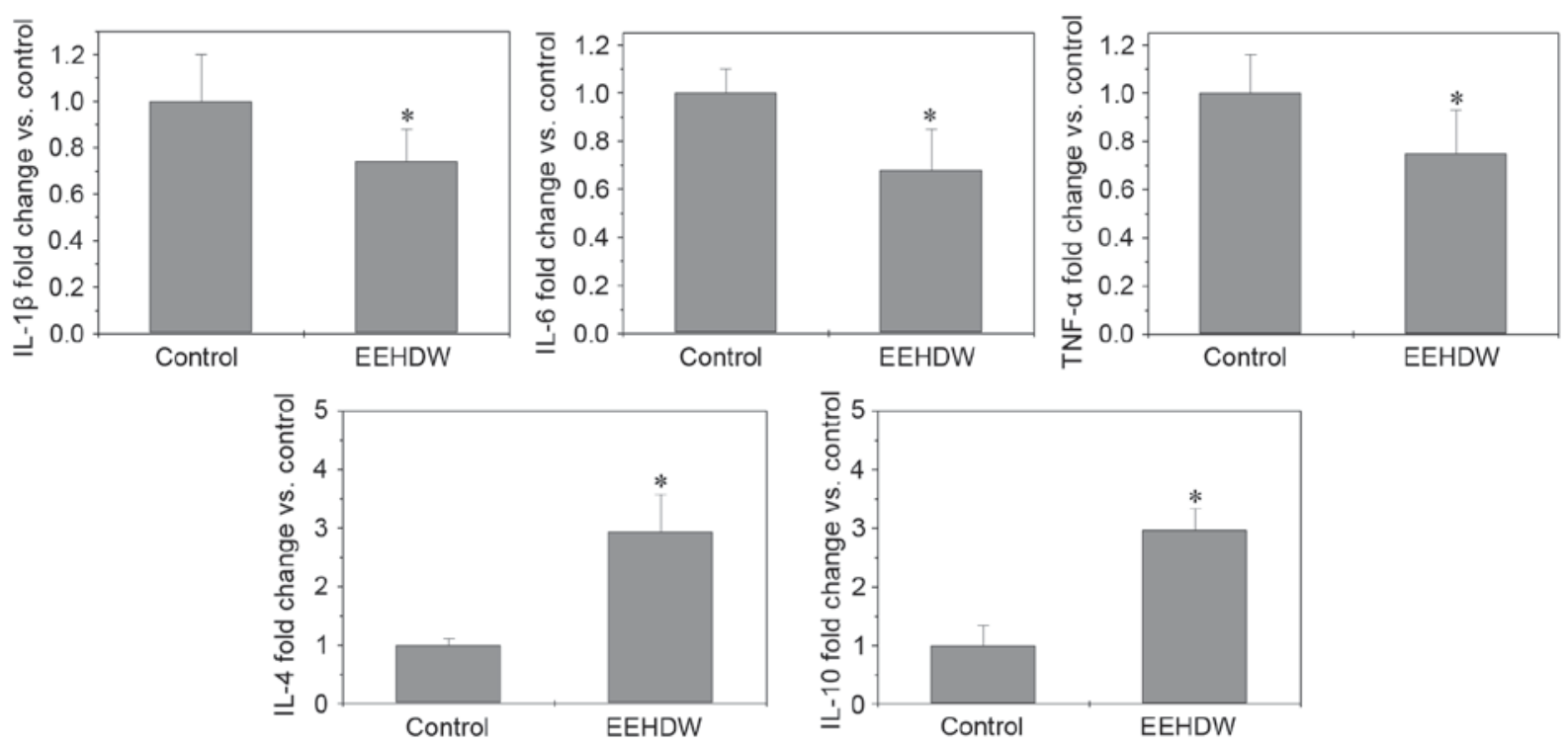

Figure 4. EEHDW regulates inflammatory cytokines in serum. The level of IL-1 $\beta$, IL-6, IL-4, IL-10 and TNF- $\alpha$ in the serum was determined by ELISA assay. The concentrations of the cytokines were determined by comparing to serial dilutions of the purified standards. Data are expressed as the mean \pm standard deviation from 10 individual mice in each group. ${ }^{*} \mathrm{P}<0.05$ vs. control mice. EEHDW, ethanol extract of Hedyotis Diffusa Willd; IL, interleukin; TNF, tumor necrosis factor.

A

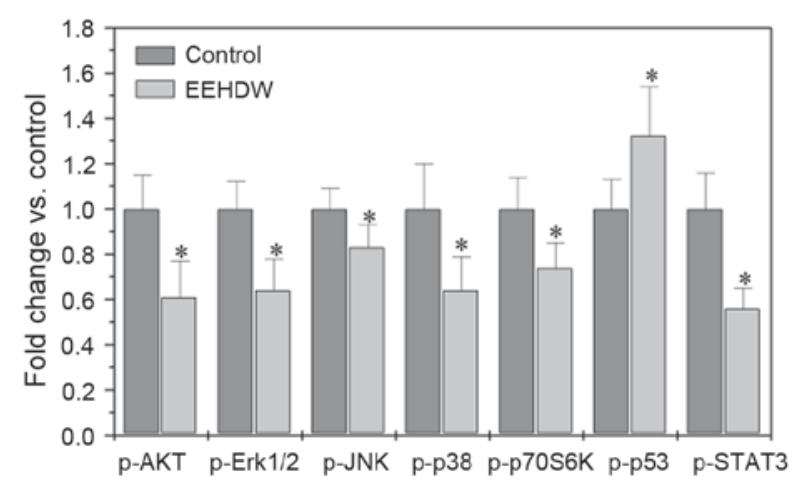

B

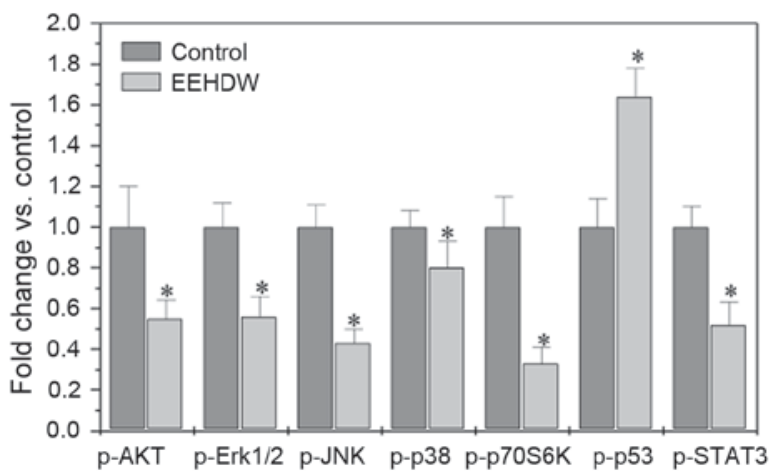

Figure 5. EEHDW regulates multiple signaling pathways in vivo and in vitro. The levels of phosphorylated AKT, Erk1/2, JNK, p38, p70S6 K, STAT3 and p53 in tumor tissues (A) or HT-29 cells (B) were determined by Bio-Plex phosphoprotein assay. The data are normalized to the levels of phosphorylated proteins in the controls. Data are expressed as the mean \pm standard deviation from 8 mice in each group or from 3 independent in vitro experiments. "P<0.05, vs. control. AKT, protein kinase B; EEHDW, ethanol extract of Hedyotis Diffusa Willd; Erk1/2, mitogen-activated protein kinase 1/2; JNK, c-Jun N-terminal kinase; p-, phosphorylated; STAT3, signal transducer and activator of transcription 3 .
p70S6K p53 and STAT3 in CRC xenograft tumor tissues and HT-29 cells was determined using Bio-Plex Phosphoprotein assay.

Treatment with EEHDW was able to significantly decrease the levels of phosphorylated AKT, Erk1/2, JNK, p38, p70S6K and STAT3 in tumor tissues $(\mathrm{P}<0.05$; Fig. 5A) and HT-29 cells compared with the control ( $\mathrm{P}<0.05$; Fig. 5B). By contrast, p-p53 expression was significantly increased following EEHDW treatment $(\mathrm{P}<0.05$; Fig. 5A and $\mathrm{B})$.

\section{Discussion}

$\mathrm{CRC}$ is a serious global health problem with high morbidity and mortality rates. To date, chemotherapy has been the main therapeutic treatment for the majority of patients with CRC (2-4). However, clinical efficacy for CRC chemotherapy remains poor due to increased drug resistance and high toxicity resulting in adverse side effects (1).

Cancer development is tightly regulated by multiple intracellular signaling pathways, including AKT, mitogen-activated protein kinase (MAPK) and STAT3 pathways, which are usually functionally redundant (6). The AKT signaling pathway is activated by factors, which induce phosphoinositide 3-kinase activation, which in turn activates the mTOR signaling pathway. The subsequent abnormal regulation of p53 and p70S6K leads to abnormalities in cell proliferation, apoptosis and differentiation. AKT signaling has been implicated in various types of cancer and is frequently implicated in anti-cancer therapeutics (28-30).

The MAPK signaling pathway is one of the main pathways involved in cell survival and proliferation. In mammals, there are three major subfamilies of MAPK, including ERK, JNK and p38. Activation of MAPK signaling is regulated by a central three-tiered kinase core, which consists of MAPK kinase kinase (MAP3K), MAPK kinase (MAP2K) and 
MAPK $(31,32)$. MAPK signaling has an important role in intestinal epithelial differentiation (33). However, aberrant activation of the MAPK signaling pathway can result in colon carcinogenesis (34-37). STAT3 is a transcription factor that exerts an essential role in cell survival and proliferation, via STAT3 activation, dimerization and subsequent nuclear translocation $(38,39)$. Constitutive activation of STAT3 is associated with the development of various types of cancer including CRC and represents poor disease prognosis (40-43). These molecular pathways modulate the expression of key genes involved in the regulation of cell proliferation, apoptosis and angiogenesis, and are therefore important in the induction, progression and metastasis of colorectal cancer (44).

The development of CRC is often characterized by complex interactions within the host immune system, ultimately resulting in the uncontrolled growth of transformed cells. For instance, chronic inflammation is an independent risk factor for the development of CRC, as well as other malignancies, including gastric cancer, hepatocellular and prostate carcinoma (45). Notably, patients who suffer from ulcerative colitis have a marked increase in the incidence of CRC (46). Experimental models of inflammation-associated colon carcinogenesis have suggested that inflammatory cell-derived cytokines can either directly or indirectly stimulate the growth of cancer cells (47-53). Nevertheless, under certain inflammatory conditions, immune cells can also mediate the anti-tumor response in order to eliminate dysplastic and cancerous cells $(54,55)$. Notably, sporadic $\mathrm{CRC}$, which represent the majority of CRC cases, exhibits extensive inflammatory infiltration with high levels of cytokine expression in the tumor microenvironment (27). The ability of immune cells to control cancer cell proliferation is mostly dependent on cytokines, which can directly target CRC cells, as well as contribute to the abnormal activation of various signaling pathways $(40,47-51)$. For example, high levels of TNF- $\alpha$ and IL-6 expression have been hypothesized to be associated with redundant activation of STAT3 pathway, which can lead to the progression of CRC (45).

Natural products, including those used in TCM, have long been used to treat multiple diseases, including cancer (17). TCM formulae consist of a combination of many natural products, each of which contains numerous chemical compounds. Therefore, TCM prescription is often considered as a multi-component and multi-target method of treatment, by exhibiting a broad range of therapeutic functions (56-62). One such prescription is EEHDW, which has exhibited specific anti-cancer activities (23).

In the present study, MTS was used to demonstrate that EEHDW reduced cell viability in different CRC cell lines in a dose- and time-dependent manner. It was also observed that EEHDW was able to significantly reduce tumor weight and volume in vivo. Furthermore, it was demonstrated that treatment with EEHDW was able to inhibit proliferation and promote apoptosis as indicated by the decrease in the percentage of Ki-67 positive cells and increasing the percentage of TUNEL positive cells. Notably, the upregulation of cytochrome c, caspase-3, caspase-9, PARP and Bax and the downregulation of Pim-1, Bcl-2, COX-2, iNOS, eNOS and HIF-1 $\alpha$ also provided further support to suggest that EEHDW is able to exert several effects on CRC, including the promotion of apoptosis, and the suppression of proliferation and angiogenesis. Although signaling pathways, including AKT, MAPK and STAT3 and cytokines such as IL-1 $\beta$, IL-6, TNF- $\alpha$, IL-4 and IL-10 displayed abnormal conditions in CRC, the present study has demonstrated that EEHDW treatment was able to alter the regulation of the multiple signaling pathways and cytokines.

In conclusion, EEHDW exerts its anti-cancer activities via altering multiple targets. Therefore, EEHDW may be developed as a promising multi-potent anti-cancer agent for the clinical treatment of CRC.

\section{Acknowledgements}

The present study was sponsored by the Research Fund for the Doctoral Program of Higher Education of China (grant no. 20133519110003) and the Developmental Fund of Chen Keji Integrative Medicine (grant nos. CKJ2014013 and CKJ2015007).

\section{References}

1. Siegel R, Miller K and Jemal A: Cancer statistics, 2016. CA Cancer J Clin 66: 7-30, 2016.

2. Ferlay J, Shin HR, Bray F, Forman D, Mathers C and Parkin DM: Estimates of worldwide burden of cancer in 2008: GLOBOCAN 2008. Int J Cancer 127: 2893-2917, 2010.

3. Center MM, Jemal A, Smith RA and Ward E: World wide variations in colorectal cancer. CA Cancer J Clin 59: 366-378, 2009.

4. Li H, Mao W, Wang X, Yu C and Du L: Incidence and mortality of cancer in Zhejiang province in 2009. Zhonghua Yu Fang Yi Xue Za Zhi 47: 592-596, 2013 (In Chinese).

5. Cunningham D, Atkin W, Lenz HJ, Lynch HT, Minsky B, Nordlinger B and Starling N: Colorectal cancer. Lancet 375: 1030-1047, 2010.

6. Jiang WQ, Fu FF, Li YX, Wang WB, Wang HH, Jiang HP and Teng LS: Molecular biomarkers of colorectal cancer: Prognostic and predictive tools for clinical practice. J Zhejiang Univ Sci B 13: 663-675, 2012.

7. Aakif M, Balfe P, Elfaedy O, Awan FN, Pretorius F, Silvio L, Castinera $\mathrm{C}$ and Mustafa H: Study on colorectal cancer presentation, treatment and follow-up. Int J Colorectal Dis 31: 1361-1363, 2016.

8. Du B and Shim JS: Targeting epithelial-mesenchymal transition (EMT) to overcome drug resistance in cancer. Molecules 21: E965, 2016.

9. Van Cutsem E, Nordlinger B and Cervantes A: Advanced colorectal cancer: ESMO clinical practice guidelines for treatment. Ann Oncol 21: 93-97, 2010.

10. Tournigand C, André T, Achille E, Lledo G, Flesh M, Mery-Mignard D, Quinaux E, Couteau C, Buyse M, Ganem G, et al: FOLFIRI followed by FOLFOX6 or the reverse sequence in advanced colorectal cancer: A randomized GERCOR study. J Clin Oncol 22: 229-237, 2004.

11. Gustin DM and Brenner DE: Chemoprevention of colon cancer: Current status and future prospects. Cancer Metastasis Rev 21: 323-348, 2002.

12. Gorlick R and Bertino JR: Drug resistance in colon cancer. Semin Oncol 26: 606-611, 1999.

13. Longley DB, Allen WL and Johnston PG: Drug resistance, predictive markers and pharmacogenomics in colorectal cancer. Biochim Biophys Acta 1766: 184-196, 2006.

14. Boose G and Stopper H: Genotoxicity of several clinically used topoisomerase II inhibitors. Toxicol Lett 116: 7-16, 2000.

15. Lin J, Chuang CC and Zuo L: Potential roles of microRNAs and ROS in colorectal cancer: Diagnostic biomarkers and therapeutic targets. Oncotarget 8: 17328-17346, 2017.

16. Ma X and Wang Z: Anticancer drug discovery in the future: An evolutionary perspective. Drug Discov Today 14: 1136-1142, 2009.

17. Yang JJ, Hsu HY, Ho YH and Lin CC: Comparative study on the immunocompetent activity of three different kinds of Peh-Hue-Juwa-Chi-Cao, Hedyotis diffusa, H. corymbosa and Mollugo pentaphylla after sublethal whole body x-irradiation. Phytother Res 11: 428-432, 1997. 
18. Song L: Zhonghuabencao. Vol 61. Shanghai Science and Technology Press, Shanghai, pp433, 1999 (In Chinese).

19. Li R, Zhao H and Lin Y: Anti-tumor effect and protective effect on chemotherapeutic damage of water soluble extracts from Hedyotis diffusa. J Chin Pharmaceu Sci 11: 54-58, 2002.

20. Lin J, Chen Y, Wei L, Chen X, Xu W, Hong Z, Sferra TJ and Peng J: Hedyotis diffusa willd extract induces apoptosis via activation of the mitochondrion-dependent pathway in human colon carcinoma cells. Int J Oncol 37: 1331-1338, 2010

21. Lin J, Wei L, Xu W, Hong Z, Liu X and Peng J: Effect of hedyotis diffusa willd extract on tumor angiogenesis. Mol Med Rep 4 1283-1288, 2011

22. Cai Q, Lin J, Wei L, Zhang L, Wang L, Zhan Y, Zeng J, Xu W, Shen A, Hong Z and Peng J: Hedyotis diffusa willd inhibits colorectal cancer growth in vivo via inhibition of STAT3 signaling pathway. Int J Mol Sci 13: 6117-28, 2012.

23. Lin M, Lin J, Wei L, Xu W, Hong Z, Cai Q, Peng J and Zhu D: Hedyotis diffusa willd extract inhibits HT-29 cell proliferation via cell cycle arrest. Exp Ther Med 4: 307-310, 2012.

24. Lin J, Wei L, Shen A, Cai Q, Xu W, Li H, Zhan Y, Hong Z and Peng J: Hedyotis diffusa willd extract suppresses sonic hedgehog signaling leading to the inhibition of colorectal cancer angiogenesis. Int J Oncol 42: 651-656, 2013.

25. Li Q, Wang X, Shen A, Zhang Y, Chen Y, Sferra TJ, Lin J and Peng J: Hedyotis diffusa willd overcomes 5 -fluorouracil resistance in human colorectal cancer HCT-8/5-FU cells by downregulating the expression of P-glycoprotein and ATP-binding casette subfamily G member 2. Exp Ther Med 10: 1845-1850, 2015.

26. Cartana ET, Gheonea DI, Cherciu IF, Streaţa I, Uscatu CD, NicoliER, Ioana M, Pirici D, Georgescu CV, Alexandru DO, et al: Assessing tumor angiogenesis in colorectal cancer by quantitative contrast-enhanced endoscopic ultrasound and molecular and immunohistochemical analysis. Endosc Ultrasound 6: 7-17, 2017.

27. Mimatsu K, Fukino N, Ogasawara Y, Saino Y and Oida T: Utility of inflammatory marker- and nutritional status-based prognostic factors for predicting the prognosis of stage iv gastric cancer patients undergoing non-curative surgery. Anticancer Res 37: 4215-4222. 2017

28. Manning BD and Cantley LC: AKT/PKB signaling: Navigating downstream. Cell 129:1261-1274, 2007.

29. Wullschleger S, Loewith R and Hall MN: TOR signaling in growth and metabolism. Cell 124:471-484, 2006.

30. Shima H, Pende M, Chen Y, Fumagalli S, Thomas G and Kozma SC: Disruption of the p70(s6k)/p85(s6k) gene reveals a small mouse phenotype and a new functional S6 kinase. EMBO J 17:6649-6659, 1998 .

31. Sebolt-Leopold JS: Development of anticancer drugs targeting the MAP kinase pathway. Oncogene 19: 6594-6599, 2000.

32. Seger R and Krebs EG: The MAPK signaling cascade. FASEB J 9: 726-735, 1995

33. Taupin D and Podolski DK: Mitogen-activated protein kinase activation regulates intestinal epithelial differentiation. Gastroenterology 116: 1072-1080, 1999.

34. Wang X, Wang Q, Hu W and Evers BM: Regulation of phorbol ester-mediated TRAF1 induction in human colon cancer cells through a PKC/RAF/ERK/NF-kappaB-dependent pathway. Oncogene 23: 1885-1895, 2004.

35. Roberts PJ and Der CJ: Targeting the Raf-MEK-ERK mitogen-activated protein kinase cascade for the treatment of cancer. Oncogene 26: 3291-3310, 2007.

36. Schwartsmann G, Di Leone LP, Dal Pizzol F and Roesler R: MAPK pathway activation in colorectal cancer: A therapeutic opportunity for GRP receptor antagonists. Lancet Oncol 6 : 444-445, 2005

37. Fang JY and Richardson BC: The MAPK signalling pathways and colorectal cancer. Lancet Oncol 6: 322-327, 2005.

38. Aggarwal BB, Kunnumakkara AB, Harikumar KB, Gupta SR, Tharakan ST, Koca C, Dey S and Sung B: Signal transducer and activator of transcription-3, inflammation and cancer: How intimate is the relationship? Ann NY Acad Sci 1171: 59-76, 2009.

39. Zhong Z, Wen Z and Darnell J: Stat3: A STAT family member activated by tyrosine phosphorylation in response to epidermal growth factor and interleukin-6. Science 264: 95-98, 1994.

40. Bromberg J and Wang TC: Inflammation and cancer: IL- 6 and STAT3 complete the link. Cancer Cell 15: 79-80, 2009.

41. Kusaba T, Nakayama T, Yamazumi K, Yakata Y, Yoshizaki A, Inoue K, Nagayasu T and Sekine I: Activation of STAT3 is a marker of poor prognosis in human colorectal cancer. Oncol Rep 15: 1445-1451, 2006
42. Lin Q, Lai R, Chirieac LR, Li C, Thomazy VA, Grammatikakis I, Rassidakis GZ, Zhang W, Fujio Y, Kunisada K, et al: Constitutive activation of JAK3/STAT3 in colon carcinoma tumors and cell lines: Inhibition of JAK3/STAT3 signaling induces apoptosis and cell cycle arrest of colon carcinoma cells. Am J Pathol 167: 969-980, 2005.

43. Xiong $\mathrm{H}$, Zhang ZG, Tian XQ, Sun DF, Liang QC, Zhang YJ, Lu R, Chen YX and Fang JY: Inhibition of JAK1, 2/STAT3 signaling induces apoptosis, cell cycle arrest and reduces tumor cell invasion in colorectal cancer cells. Neoplasia 10: 287-297, 2008.

44. García-Caballero M, Paupert J, Blacher S, Van de Velde M, Quesada AR, Medina MA and Noël A: Targeting VEGFR-3/-2 signaling pathways with AD0157: A potential strategy against tumor-associated lymphangiogenesis and lymphatic metastases. J Hematol Oncol 10: 122, 2017.

45. Fearon ER and Vogelstein B: A genetic model for colorectal tumorigenesis. Cell 61: 759-767, 1990.

46. Eaden JA, Abrams KR and Mayberry JF: The risk of colorectal cancer in ulcerative colitis: A meta-analysis. Gut 48: 526-535, 2001.

47. Grivennikov S, Karin E, Terzic J, Mucida D, Yu GY, Vallabhapurapu S, Scheller J, Rose-John S, Cheroutre H, Eckmann L and Karin M: IL-6 and Stat 3 are required for survival of intestinal epithelial cells and development of colitis-associated cancer. Cancer Cell 15: 103-113, 2009.

48. Huber S, Gagliani N, Zenewicz LA, Huber FJ, Bosurgi L, Hu B, Hedl M, Zhang W, O'Connor W Jr, Murphy AJ, et al: IL-22BP is regulated by the inflammasome and modulates tumorigenesis in the intestine. Nature 491: 259-263, 2012

49. Hyun YS, Han DS, Lee AR, Eun CS, Youn J and Kim HY: Role of IL-17A in the development of colitis-associated cancer. Carcinogenesis 33: 931-936, 2012.

50. Jauch D, Martin M, Schiechl G, Kesselring R, Schlitt HJ, Geissler EK and Fichtner-Feigl S: Interleukin 21 controls tumour growth and tumour immunosurveillance in colitis-associatedtumorigenesis in mice. Gut 60: 1678-1686, 2011.

51. Kirchberger S, Royston DJ, Boulard O, Thornton E, Franchini F, Szabady RL, Harrison O and Powrie F: Innate lymphoid cells sustain colon cancer through production of interleukin-22 in a mouse model. J Exp Med 210: 917-931, 2013.

52. Popivanova BK, Kitamura K, Wu Y, Kondo T, Kagaya T, Kaneko S, Oshima M, Fujii C and Mukaida N: Blocking TNF-alpha in mice reduces colorectal carcinogenesis associated with chronic colitis. J Clin Invest 118: 560-570, 2008.

53. Stolfi C, Rizzo A, Franzè E, Rotondi A, Fantini MC, Sarra M, Caruso R, Monteleone I, Sileri P, Franceschilli L, et al: Involvement of interleukin-21 in the regulation of colitis-associated colon cancer. J Exp Med 208: 2279-2290, 2011

54. Lanca T and Silva-Santos B: The split nature of tumor-infiltrating leukocytes: Implicationsfor cancer surveillance and immunotherapy. Oncoimmunology 1: 717-725, 2012

55. Monteleone G, Pallone F and Stolfi C: The dual role of inflammation in colon carcinogenesis. Int J Mol Sci 13: 11071-11084, 2012.

56. Newman DJ, Cragg GM and Snader KM: The influence of natura products upon drug discovery. Nat Prod Rep 17: 215-234, 2000.

57. Gordaliza M: Natural products as leads to anticancer drugs. Clin Transl Oncol 9: 767-776, 2007.

58. Peng J, Chen Y, Lin J, Zhuang Q, Xu W, Hong Z and Sferra TJ: Patrinia scabiosaefolia extract suppresses proliferation and promotes apoptosis by inhibiting STAT3 pathway in human multiple myeloma cells. Mol Med Rep 4: 313-318, 2011.

59. Cai Q, Lin J, Wei L, Zhang L, Wang L, Zhan Y, Zeng J, Xu W, Shen A, Hong $Z$ and Peng J: Hedyotis diffusa Willd inhibits colorectal cancer growth in vivo via inhibition of STAT3 signaling pathway. Int J Mol Sci 13: 6117-6128, 2012.

60. Wei L, Chen Y, Lin J, Zhao Y, Chen X, Xu W, Liu X, Sferra TJ and Peng J: Scutellaria barbata D. don induces apoptosis of human colon carcinoma cell through activation of the mitochondrion-dependent pathway. J Med Plants Res 5: 1962-1970, 2011.

61. Wei L, Lin J, Xu W, Hong Z, Liu X, Sferra TJ and Peng J: Inhibition of tumor angiogenesis by scutellaria barbata D. don via suppressing proliferation, migration and tube formation of endothelial cells and downregulation of the expression of VEGF-A in cancer cells. J Med Plants Res 5: 3260-3268, 2011.

62. Zheng L, Chen Y, Lin W, Zhuang Q, Chen X, Xu W, Liu X, Peng J and Sferra TJ: Spica Prunellae extract promotes mitochondrion-dependent apoptosis in a human colon carcinoma cell line. Afr J Pharm Pharmacol 5: 327-335, 2011. 